\title{
Testing the blazar sequence and black hole mass scaling with BL Lac objects
}

\author{
Richard M. Plotkin ${ }^{1}$, Sera Markoff ${ }^{1}$, Scott F. Anderson ${ }^{2}$, \\ Brandon C. Kelly ${ }^{3}$, Elmar Körding ${ }^{4}$, and Scott C. Trager ${ }^{5}$ \\ ${ }^{1}$ Astronomical Institute "Anton Pannekoek", University of Amstserdam, \\ Science Park 904, 1098 XH, Amsterdam, the Netherlands \\ email: r.m.potkin@uva.nl \\ ${ }^{2}$ Dept. of Astronomy, University of Washington, \\ Box 351580 , Seattle, WA 98195, USA \\ ${ }^{3}$ Harvard-Smithsonian Center for Astrophysics, \\ 60 Garden St., Cambridge, MA 02138, USA \\ ${ }^{4}$ Université Paris Diderot and Service d'Astrophysique, UMR AIM, \\ CEA Saclay, F-91191 Gif-sur-Yvette, France \\ ${ }^{5}$ Kapteyn Astronomical Institute, University of Groningen, \\ Postbus 800, NL-9700 AV Groningen, the Netherlands
}

\begin{abstract}
Jets from accreting black holes appear remarkably similar over eight orders of magnitude in black hole mass, with more massive black holes generally launching more powerful jets. For example, there is an observed correlation, termed the fundamental plane of black hole accretion, between black hole mass, radio luminosity, and X-ray luminosity. Here, we probe the high-mass tail $\left(10^{8}-10^{9} M_{\odot}\right)$ of the accreting black hole distribution with BL Lac objects. We build SEDs for hundreds of SDSS BL Lacs, and we use these SEDs to test the blazar sequence, a proposed anti-correlation between jet power and peak frequency. We then show our BL Lacs fit on the fundamental plane, supporting the non-linear scaling of jet radiation with black hole mass. The subset of BL Lacs considered here compose the largest sample yet used in the above types of studies, reducing potential selection effects and biases.
\end{abstract}

Keywords. galaxies: active, BL Lacertae objects: general, galaxies: jets

BL Lac objects are a subset of blazars unified with low-luminosity radio galaxies viewed nearly along the axis of a relativistic jet. Their spectral energy distributions (SEDs) are dominated by beamed jet emission, yielding synchrotron radiation peaking anywhere from the near-infrared to the soft X-ray, and inverse Compton emission at higher frequencies. Here, we use BL Lacs to shed light on two properties of relativistic jets. First, we investigate if their SED shape depends on luminosity (the blazar sequence), and then we test the scaling of jet radiation with black hole mass. We use a large uniformly selected BL Lac sample from the SDSS (Plotkin et al. 2010, hereafter P10), and we build SEDs using FIRST, NVSS, WENSS, and GB6 in the radio, 2MASS in the near-infrared, GALEX in the ultraviolet, and RASS and the XMM-Newton Slew Survey in the X-ray.

The Blazar Sequence: The blazar sequence was discovered by Fossati et al. (1998), and it has so far been supported by the Fermi Gamma-Ray Observatory. We test the blazar sequence by fitting parabolas to 409 SDSS BL Lacs with amply populated SEDs, and we then compare radio luminosity with peak frequency (Figure 1a). Only a very weak anti-correlation is observed (green solid line), with significance $\mathrm{p}=0.00425$. However, if we only consider the 30 brightest radio sources and the 30 brightest X-ray sources to simulate the BL Lacs included in Fossati et al. (1998), then the anti-correlation becomes quite significant $\left(\mathrm{p}<10^{-5}\right.$, orange dashed line). We thus conclude deep blazar surveys 

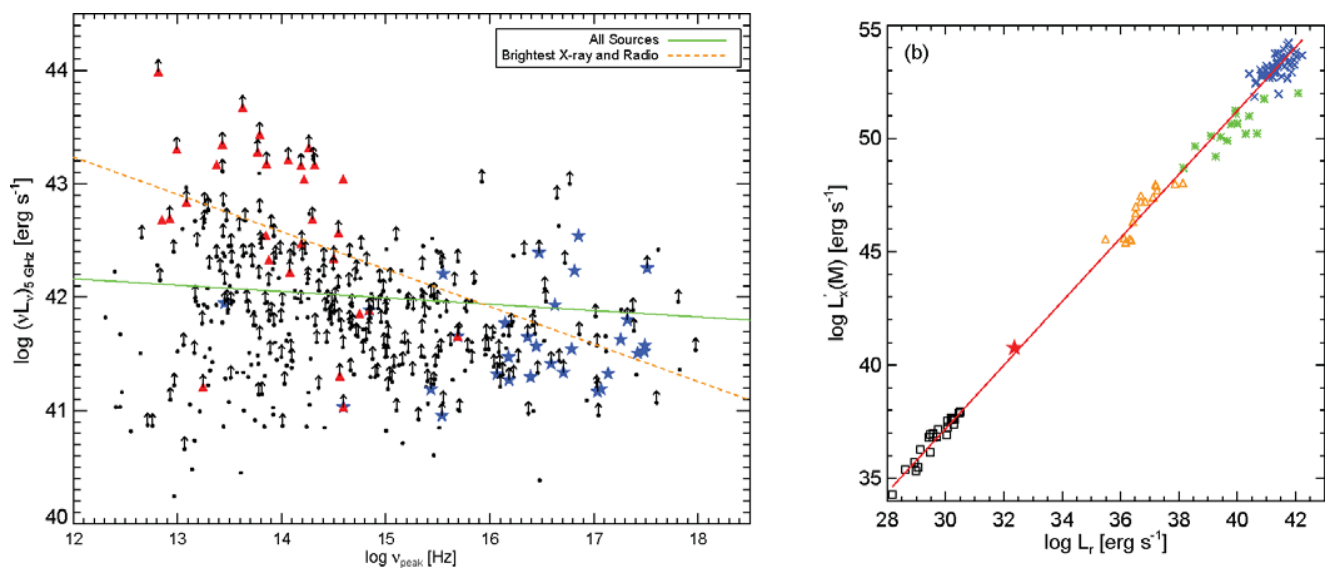

Figure 1. (a) Logarithm of rest-frame $5 \mathrm{GHz}$ radio luminosity vs. logarithm of synchrotron peak frequency for $409 \mathrm{BL}$ Lacs. The 30 brightest radio and 30 brightest X-ray sources are shown as red triangles and blue stars, respectively. Redshift (and therefore luminosity) lower limits are estimated if necessary from the fact we do not detect host galaxy emission (see P10). (b) The fundamental plane: log X-ray luminosity (normalized by black hole mass) vs. $\log$ radio luminosity. Included are X-ray binaries $\left(\sim 10 M_{\odot}\right.$, black squares), Sgr $\mathrm{A}^{*}$ $\left(\sim 10^{6} M_{\odot}\right.$, red star $)$, LLAGN $\left(\sim 10^{7-8} M_{\odot}\right.$, orange triangles $)$, FR I $\left(\sim 10^{8-9} M_{\odot}\right.$, green asterisks), and BL Lacs $\left(\sim 10^{8-9} M_{\odot}\right.$, blue crosses). The FR I and BL Lac X-ray luminosities are extrapolated from the luminosity where the jet becomes optically thin (assuming $f_{\nu} \sim \nu^{-0.6}$ ), otherwise radiative losses from synchrotron cooling become important. We find $\log L_{X}=(1.40 \pm 0.04) \log L_{r}-(0.87 \pm 0.07) \log M_{b h}-(4.89 \pm 1.10)$ for our 113 objects.

reveal low-luminosity low-energy peaked BL Lacs that weaken the blazar sequence. We will explore this further by fitting our BL Lac SEDs with models more physical than a parabola (including multi-zone synchrotron and synchrotron self-compton).

Black Hole Mass Scaling: The fundamental plane is one of the strongest arguments supporting mass scaling (Merloni et al. 2003, Falcke et al. 2004). We use 58 data points from the KFC sample in Körding et al. (2006), and we replace their BL Lacs with 55 from P10 with central black hole mass estimates (Plotkin et al. 2010, subm.). Our motivation for including BL Lacs is we know their X-ray emission comes from a jet and not a corona (the objects in the KFC sample are also likely jet dominated). Using the Bayesian regression technique of Kelly (2007), we find $L_{X} \sim L_{r}^{1.40} M_{b h}^{-0.87}$ (Figure 1b, the regression is similar if we debeam the BL Lacs). This is consistent with the theoretical prediction of Falcke et al. (2004) for jet dominated systems. Merloni et al. (2003), however, find a different regression $\left(L_{X} \sim L_{r}^{1.64} M_{b h}^{-1.30}\right)$ because their sample includes luminous X-ray binaries and AGN (i.e., hard X-rays are rather emitted by a corona). This study thus supports the notion that mass scaling investigations must compare black holes in similar accreting "states." This work was supported by a Netherlands Organization for Scientific Research (NWO) Vidi Fellowship and NASA grant NNX09AF89G.

\section{References}

Falcke, H., Körding, E., \& Markoff, S. 2004, A\&A, 414, 895

Fossati, G., Maraschi, L., Celotti, A., Comastri, A., \& Ghisellini, G. 1998, MNRAS, 299, 433

Kelly, B. C. 2007, ApJ, 665, 1489

Körding, E., Falcke, H., \& Corbel, S. 2006 A\&A, 456, 439

Merloni, A., Heinz, S., \& di Matteo, T. 2003, MNRAS, 345, 1057

Plotkin, R. M., et al. 2010 AJ, 139, 390 (P10) 